\title{
On the topological properties of the generalized Clarke subdifferential
}

\author{
S. Lahrech
}




\title{
ON THE TOPOLOGICAL PROPERTIES OF THE GENERALIZED CLARKE SUBDIFFERENTIAL
}

\author{
S. LAHRECH \\ Dedicated to the memory of S. Bach.
}

Received 26 September, 2005

\begin{abstract}
We give some topological properties of the generalized Clarke subdifferential which is an extension of the notion of the Clarke subdifferentiability [5]. Potential advantages of generalized Clarke subdifferential over other concepts of subdifferentiability might be related to the fact that it offers a rich calculus and applications. It allows us, for example, to present a convenient test for the weak metric regularity of mappings non-necessarily strictly Fréchet differentiable.
\end{abstract}

2000 Mathematics Subject Classification: 49J52, 49J50

Keywords: generalized Clarke subdifferential, Clarke subdifferential, strictly Taylor differentiable mappings, strictly Hadamard differentiable mappings

\section{AN INTRODUCTION TO GENERALIZED CLARKE SUBDIFFERENTIABILITY}

We shall be working in binormed space $\left(E,\|\cdot\|_{1},\|\cdot\|_{2}\right)$ such that $\left(E,\|\cdot\|_{2}\right)$ is a Banach space and, for some $c>0$, the condition $\|\cdot\|_{1} \leq c\|\cdot\|_{2}$ holds.

Let $U$ be an open set of $\left(E,\|\cdot\|_{1}\right)$, and let $h_{2}$ be a $\|\cdot\|_{2}$ locally Lipschitz real function around $\bar{x} \in U$.

Let $v$ any vector in $E$. The Clarke generalized directional derivative of $h_{2}$ at $\bar{x}$ in the direction $v$ with respect to the norm $\|\cdot\|_{2}$, denoted $h_{2}^{0,2}(\bar{x}, v)$, is defined as follows:

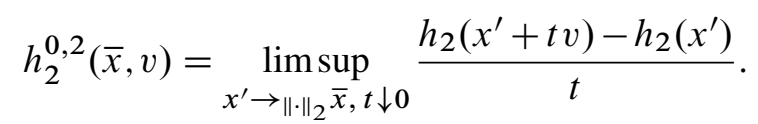

The Clarke subdifferential of $h_{2}$ at $\bar{x}$ with respect to the norm $\|\cdot\|_{2}$, denoted $\partial_{0}^{2} h_{2}(\bar{x})$, is the subset of $\left(E,\|\cdot\|_{2}\right)^{\prime}$ given by

$$
\partial_{0}^{2} h_{2}(\bar{x})=\left\{\xi \in\left(E,\|\cdot\|_{2}\right)^{\prime}: h_{2}^{0,2}(\bar{x}, v) \geq\langle\xi, v\rangle \forall v \in E\right\} .
$$

According to [5], the generalized Clarke subdifferential of $h_{2}$ at $\bar{x}$ with respect to the pair of norms $\left(\|\cdot\|_{1},\|\cdot\|_{2}\right)$, denoted $\partial_{0}^{1,2} h_{2}(\bar{x})$, is the subset of $\left(E,\|\cdot\|_{2}\right)^{\prime}$ given by

$$
\partial_{0}^{1,2} h_{2}(\bar{x})=\left\{\xi \in\left(E,\|\cdot\|_{2}\right)^{\prime}: h_{2}^{0,1}(\bar{x}, v) \geq\langle\xi, v\rangle \forall v \in E\right\} .
$$


Notice that the Clarke subdifferential $\partial_{0}^{2} h_{2}(\bar{x})$ is smaller than the generalized Clarke subdifferential $\partial_{0}^{1,2} h_{2}(\bar{x})$. More precisely, we have $\partial_{0}^{2} h_{2}(\bar{x}) \subset \partial_{0}^{1,2} h_{2}(\bar{x})$ and also $\partial_{0}^{1} h_{2}(\bar{x}) \subset \partial_{0}^{1,2} h_{2}(\bar{x})$.

If moreover, $h_{2}$ is locally Lipschitz around $\bar{x}$ with respect to the norm $\|\cdot\|_{1}$, then $\partial_{0}^{2} h_{2}(\bar{x})=\partial_{0}^{1} h_{2}(\bar{x})=\partial_{0}^{1,2} h_{2}(\bar{x})$.

In particular, if we take $\|\cdot\|_{1}=\|\cdot\|_{2}$, then $\partial_{0}^{2} h_{2}(\bar{x})=\partial_{0}^{1} h_{2}(\bar{x})=\partial_{0}^{1,2} h_{2}(\bar{x})$.

Thus, the generalized Clarke subdifferential is a generalized version of the Clarke subdifferential.

Let us note that $h_{2}^{0,1}(\bar{x}, \cdot)$ is the support function of the generalized Clarke subdifferential $\partial_{0}^{1,2} h_{2}(\bar{x})$ viewed as subset of $\left(E,\|\cdot\|_{2}\right)^{\prime}$. Finally, note that even if $h_{2}^{0,1}(\bar{x},$.$) is the support function of the Clarke subdifferential \partial_{0}^{1} h_{2}(\bar{x})$, the generalized Clarke subdifferential $\partial_{0}^{1,2} h_{2}(\bar{x})$ does not necessarily coincide with $\partial_{0}^{1} h_{2}(\bar{x})$, because $\partial_{0}^{1} h_{2}(\bar{x})$ is not, in general, weak ${ }^{*}$-closed in $\left(\left(E,\|\cdot\|_{2}\right)^{\prime}, * \sigma\left(\left(E,\|\cdot\|_{2}\right)^{\prime}, E\right)\right)$.

We refer to [1-6] for more details on the notions mentioned.

\section{BASIC PROPERTIES OF GENERALIZED CLARKE SUBDIFFERENTIAL}

Assume that all the hypothesis of the above paragraph are satisfied.

\subsection{Topological properties of generalized Clarke subdifferential}

Our goals in this paragraph is to give some topological properties of generalized Clarke subdifferential. For this, we introduce the notion of locally Lipschitzian mappings with respect to the pair of norms.

We say that $h_{2}$ is $\left(\|\cdot\|_{1},\|\cdot\|_{2}\right)$ locally Lipschitzian around $\bar{x}$ if for some nonnegative scalar $K$, one has

$$
\left|h_{2}(y)-h_{2}\left(y^{\prime}\right)\right| \leq K\left\|y-y^{\prime}\right\|_{2}
$$

for all $y, y^{\prime}$ close to $\bar{x}$ with respect to the norm $\|\cdot\|_{1}$.

The following summarizes some basic properties of the generalized Clarke subdifferential.

Proposition 1. Let $\partial_{0}^{1,2} h_{2}(\bar{x})$ be a nonempty, convex, closed subset of the space $\left(\left(E,\|\cdot\|_{2}\right)^{\prime}, * \sigma\left(\left(E,\|\cdot\|_{2}\right)^{\prime}, E\right)\right)$. Moreover, if $h_{2}$ is $\left(\|\cdot\|_{1},\|\cdot\|_{2}\right)$ locally Lipschitzian around $\bar{x}$, then $\partial_{0}^{1,2} h_{2}(\bar{x})$ is weakly*-compact in $\left(\left(E,\|\cdot\|_{2}\right)^{\prime}, * \sigma\left(\left(E,\|\cdot\|_{2}\right)^{\prime}, E\right)\right)$.

Proof. The set $\partial_{0}^{1,2} h_{2}(\bar{x})$ is nonempty, because $\partial_{0}^{2} h_{2}(\bar{x}) \subset \partial_{0}^{1,2} h_{2}(\bar{x})$. The convexity and the closeness of $\partial_{0}^{1,2} h_{2}(\bar{x})$ are trivially fulfilled.

Assume now that $h_{2}$ is $\left(\|\cdot\|_{1},\|\cdot\|_{2}\right)$ locally Lipschitzian around $\bar{x}$. Then, $\partial_{0}^{1,2} h_{2}(\bar{x})$ is bounded in $\left(\left(E,\|\cdot\|_{2}\right)^{\prime},\|\cdot\|_{\left(E,\|\cdot\|_{2}\right)^{\prime}}\right)$. Therefore, using the Alaoglu theorem, we deduce the weak*-compactness of $\partial_{0}^{1,2} h_{2}(\bar{x})$.

The assertion below reiterates that the pair of multifunctions $\left(\partial_{0}^{2} h_{2}, \partial_{0}^{1,2} h_{2}\right)$ is closed from $\left(U,\|\cdot\|_{1}\right)$ to $\left(\left(E,\|\cdot\|_{2}\right)^{\prime}, * \sigma\left(\left(E,\|\cdot\|_{2}\right)^{\prime}, E\right)\right)$ in the following sense: if 
$x_{i}$ and $\xi_{i}$ are sequences in $U$ and $\left(E,\|\cdot\|_{2}\right)^{\prime}$ such that $\xi_{i} \in \partial_{0}^{2} h_{2}\left(x_{i}\right), x_{i}$ converges to $x$ with respect to the norm $\|\cdot\|_{1}$, and $\xi_{i}$ converges to $\xi$ in the weak topology $* \sigma\left(\left(E,\|\cdot\|_{2}\right)^{\prime}, E\right)$, then $\xi \in \partial_{0}^{1,2} h_{2}(x)$.

Proposition 2. The pair of multifunctions $\left(\partial_{0}^{2} h_{2}, \partial_{0}^{1,2} h_{2}\right)$ is closed from $\left(U,\|\cdot\|_{1}\right)$ to $\left(\left(E,\|\cdot\|_{2}\right)^{\prime}, * \sigma\left(\left(E,\|\cdot\|_{2}\right)^{\prime}, E\right)\right)$.

Proof. Let $x_{i}$ and $\xi_{i}$ be sequences in $U$ and $\left(E,\|\cdot\|_{2}\right)^{\prime}$ such that $\xi_{i} \in \partial_{0}^{2} h_{2}\left(x_{i}\right)$, $x_{i}$ converges to $x$ with respect to the norm $\|\cdot\|_{1}$, and $\xi_{i}$ converges to $\xi$ in the weak topology $* \sigma\left(\left(E,\|\cdot\|_{2}\right)^{\prime}, E\right)$.

Let any $v \in E$ be given. Then $\left\langle\xi_{i}, v\right\rangle$ converges to $\langle\xi, v\rangle$. One has $h_{2}^{0,2}\left(x_{i}, v\right) \geq$ $\left\langle\xi_{i}, v\right\rangle$, which implies that $h_{2}^{0,1}\left(x_{i}, v\right) \geq\left\langle\xi_{i}, v\right\rangle$.

By the upper semicontinuity of $h_{2}^{0,1}$ with respect to the norm $\|\cdot\|_{1}$, we deduce that $h_{2}^{0,1}(x, v) \geq\langle\xi, v\rangle$. Since $v$ is arbitrary, $\xi$ belongs to $\partial_{0}^{1,2} h_{2}(x)$.

Remark 1. If $h_{2}$ is $\left(\|\cdot\|_{1},\|\cdot\|_{2}\right)$ locally Lipschitzian around $\bar{x}$. Then, $\zeta \in \partial_{0}^{1,2} h_{2}(\bar{x})$ if and only if $h_{2}^{0,1}(\bar{x}, v) \geq\langle\zeta, v\rangle \forall v \in E$.

\subsection{Relation to Taylor derivatives and generalized subderivatives}

The main result of this section will be that if $h_{2}$ is $\left(\|\cdot\|_{1},\|\cdot\|_{2}\right)$ locally Lipschitzian around $\bar{x}$, then $\partial_{0}^{1,2} h_{2}(\bar{x})$ is reduced to a singleton if and only if $h_{2}$ is $\left(\|\cdot\|_{1},\|\cdot\|_{2}\right)$ strictly Taylor differentiable at $\bar{x}$.

By $B_{1}$, respectively, $B_{2}$, we denote the unit ball in $\left(E,\|\cdot\|_{1}\right)$, respectively, the unit ball in $\left(E,\|\cdot\|_{2}\right)$.

Let $h$ be a map acting from $U$ into a Banach space $(Y,\|\cdot\|)$, and let $x \in U$. We shall say that $h$ is $\left(\|\cdot\|_{1},\|\cdot\|_{2}\right)$ strictly Taylor differentiable at a point $x$ if there exists a continuous linear operator from $\left(E,\|\cdot\|_{2}\right)$ to $(Y,\|\cdot\|)$ denoted $\nabla h(x)$ such that for each $v$, the condition

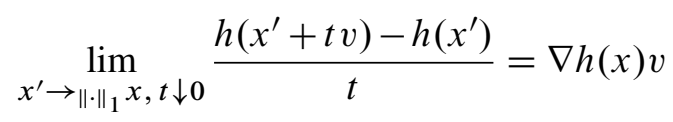

holds, where the convergence is uniform for $v$ in compact sets in $\left(E,\|\cdot\|_{2}\right)$. Note that ours is a Hadamard-type strict derivative.

Let us remark that if $h$ is a $\left(\|\cdot\|_{1},\|\cdot\|_{2}\right)$ strictly Taylor differentiable at $x$, then $h$ is not necessarily $\|\cdot\|_{1}$ strictly differentiable at $x$. But if $\|\cdot\|_{1}$ is equivalent to the norm $\|\cdot\|_{2}$, then $h$ is necessarily $\|\cdot\|_{1}$-strictly differentiable at $x$.

So the notion of strict differentiability in terms of Taylor strengthens and generalizes the elegant notion of Hadamard-strict differentiability.

Proposition 3. Let $L$ be a continuous linear operator from $\left(E,\|\cdot\|_{2}\right)$ to $(Y,\|\cdot\|)$, and let $x \in U$. The following assertions are equivalent:

(i) $h$ is $\left(\|\cdot\|_{1},\|\cdot\|_{2}\right)$ strictly Taylor differentiable at $x$ and $\nabla h(x)=L$; 
(ii) $h$ is $\left(\|\cdot\|_{1},\|\cdot\|_{2}\right)$ locally Lipschitzian around $x$, and for each $v$ in $E$ one has

$$
\lim _{x^{\prime} \rightarrow\|\cdot\|_{1} x, t \downarrow 0} \frac{h\left(x^{\prime}+t v\right)-h\left(x^{\prime}\right)}{t}=L v .
$$

Proof. Assume (i). The equality in (ii) holds by assumption, so to prove (ii) it suffices to show that $h$ is $\left(\|\cdot\|_{1},\|\cdot\|_{2}\right)$ locally Lipschitzian around $x$. If this is not the case, there exist sequences $\left\{x_{n}\right\}$ and $\left\{x_{n}^{\prime}\right\}$ converging to $x$ with respect to the norm $\|\cdot\|_{1}$ such that $x_{n}$ and $x_{n}^{\prime}$ lie in $x+\frac{1}{n} B_{1}$ and

$$
\left\|h\left(x_{n}\right)-h\left(x_{n}^{\prime}\right)\right\|>n\left\|x_{n}-x_{n}^{\prime}\right\|_{2} .
$$

Let us define $t_{n}>0$ and $v_{n}$ via $x_{n}^{\prime}=x_{n}+t_{n} v_{n}$ and $\left\|v_{n}\right\|_{2}=n^{-\frac{1}{2}}$. It follows that $t_{n} \rightarrow 0$.

Let $V$ consists of the points in the sequence $\left\{v_{n}\right\}$ together with 0 . Note that $V$ is compact in $\left(E,\|\cdot\|_{2}\right)$, so that by definition of $\nabla h(x)$ for any $\varepsilon$ there exists $n_{0}$ such that, for all $n \geq n_{0}$, for all $v \in V$, one has

$$
\left\|\frac{h\left(x_{n}+t_{n} v\right)-h\left(x_{n}\right)}{t_{n}}-\nabla h(x) v\right\|<\varepsilon .
$$

But this is impossible since when $v=v_{n}$, the term $t_{n}{ }^{-1}\left(h\left(x_{n}+t_{n} v\right)-h\left(x_{n}\right)\right)$ has norm exceeding $n^{\frac{1}{2}}$ by construction. Thus, (ii) holds.

We now posit (ii). Let $V$ any compact subset of $\left(E,\|\cdot\|_{2}\right)$ and $\varepsilon$ any positive number. In view of (ii), there exists for each $v$ in $V$ a number $\delta(v)$ such that

$$
\left\|\frac{h\left(x^{\prime}+t v\right)-h\left(x^{\prime}\right)}{t}-\nabla h(x) v\right\|<\varepsilon
$$

for all $x^{\prime} \in x+\delta(v) B_{1}$ and $\left.t \in\right] 0, \delta[$,. Since the norm of

$$
\frac{h\left(x^{\prime}+t v^{\prime}\right)-h\left(x^{\prime}\right)}{t}-\frac{h\left(x^{\prime}+t v\right)-h\left(x^{\prime}\right)}{t}
$$

is bounded above by $k\left\|v-v^{\prime}\right\|_{2}$ (where $K$ is a $\left(\|\cdot\|_{1},\|\cdot\|_{2}\right.$ ) Lipschitz constant for $h$ and where $x^{\prime}$ is sufficiently $\|\cdot\|_{1}$ near $x$ and $t$ is sufficiently near 0 ), we deduce from the last inequality that for a suitable redefinition of $\delta(v)$, one has

$$
\left\|\frac{h\left(x^{\prime}+t v^{\prime}\right)-h\left(x^{\prime}\right)}{t}-L v^{\prime}\right\|<2 \varepsilon
$$

for all $x^{\prime}$ in $x+\delta(v) B_{1}, v^{\prime}$ in $v+\delta(v) B_{2}$, and $t$ in $] 0, \delta\left[\right.$. A finite number of the $\|\cdot\|_{2^{-}}$ open sets $\left\{v+\delta(v) B_{2}: v \in V\right\}$ will cover $V$, say, those that correspond to $v_{1}, \ldots, v_{n}$. If we set $\delta^{\prime}=\min _{1 \leq i \leq n} \delta\left(v_{i}\right)$, it follows then that

$$
\left\|\frac{h\left(x^{\prime}+t v^{\prime}\right)-h\left(x^{\prime}\right)}{t}-L v^{\prime}\right\|<2 \varepsilon
$$

for any $v \in V$, for all $x^{\prime} \in x+\delta^{\prime} B_{1}$ and $\left.t \in\right] 0, \delta^{\prime}\left[\right.$. Thus, $h$ is $\left(\|\cdot\|_{1},\|\cdot\|_{2}\right)$ strictly Taylor differentiable at $x$ and $\nabla h(x)=L$, and the proof is complete. 
Now we can prove the main result of this section.

Theorem 1. Assume that $h_{2}$ is $\left(\|\cdot\|_{1},\|\cdot\|_{2}\right)$ locally Lipschitzian around $\bar{x}$. Then $\partial_{0}^{1,2} h_{2}(\bar{x})$ is reduced to a singleton $\{\zeta\}$ if and only if $h_{2}$ is $\left(\|\cdot\|_{1},\|\cdot\|_{2}\right)$ strictly Taylor differentiable at $\bar{x}$. In this case, $\zeta=\nabla h_{2}(\bar{x})$.

In particular, if $\|\cdot\|_{1}=\|\cdot\|_{2}$ then $\partial_{0}^{1} h_{2}(\bar{x})$ is reduced to a singleton $\{\zeta\}$ if and only if $h_{2}$ is $\|\cdot\|_{1}$ strictly differentiable at $\bar{x}$.

Proof. Suppose first that $h_{2}$ is $\left(\|\cdot\|_{1},\|\cdot\|_{2}\right)$ strictly Taylor differentiable at $\bar{x}$, then $h_{2}^{0,1}(\bar{x}, v)=\nabla h_{2}(\bar{x}) v$. Therefore, $\partial_{0}^{1,2} h_{2}(\bar{x})$ is reduced to a singleton $\left\{\nabla h_{2}(\bar{x})\right\}$.

To prove the converse, it suffices to show that the condition of Proposition 3 holds if $\partial_{0}^{1,2} h_{2}(\bar{x})$ is reduced to a singleton $\{\zeta\}$. We begin by showing that $h_{2}^{0,1}(\bar{x}, v)=$ $\langle\zeta, v\rangle$ for each $v$.

Let $v \in E$ (note that $h_{2}^{0,1}(\bar{x}, v) \geq\langle\zeta, v\rangle$ ). By the Hahn-Banach theorem there exists $\zeta^{\prime} \in\left(E,\|\cdot\|_{1}\right)^{\prime}$ majorized by $h_{2}^{0,1}(\bar{x}, \cdot)$ and agreeing with $h_{2}^{0,1}(\bar{x}, \cdot)$ at $v$. Since $h_{2}$ is $\left(\|\cdot\|_{1},\|\cdot\|_{2}\right)$ locally Lipschitzian around $\bar{x}$, then $\zeta^{\prime} \in\left(E,\|\cdot\|_{2}\right)^{\prime}$. It follows then that $\zeta^{\prime} \in \partial_{0}^{1,2} h_{2}(\bar{x})$. Consequently, $\zeta=\zeta^{\prime}$. Therefore, $h_{2}^{0,1}(\bar{x}, v)=\langle\zeta, v\rangle$.

We now calculate

$$
\begin{aligned}
\liminf _{x^{\prime} \rightarrow\|\cdot\|_{1} \bar{x}, t \downarrow 0} \frac{h_{2}\left(x^{\prime}+t v\right)-h_{2}\left(x^{\prime}\right)}{t} & =-\limsup _{x^{\prime} \rightarrow\left\|_{1} \cdot\right\|_{1}, t \downarrow 0} \frac{h_{2}\left(x^{\prime}\right)-h_{2}\left(x^{\prime}+t v\right)}{t} \\
& =-\limsup _{x^{\prime} \rightarrow \|_{1} \bar{x}, t \downarrow 0} \frac{h_{2}\left(x^{\prime}+t v-t v\right)-h_{2}\left(x^{\prime}+t v\right)}{t} \\
& =-h_{2}^{0,1}(\bar{x},-v) \\
& =-\langle\zeta,-v\rangle \\
& =\langle\zeta, v\rangle \\
& =h_{2}^{0,1}(\bar{x}, v) \\
& =\limsup _{x^{\prime} \rightarrow\left\|_{1}\right\|_{1}, t \downarrow 0} \frac{h_{2}\left(x^{\prime}+t v\right)-h_{2}\left(x^{\prime}\right)}{t} .
\end{aligned}
$$

This establishes the limit condition of Proposition 3 and completes the proof.

Example 1. Let $\Omega$ a bounded domain in $R^{2}, E=W_{0}^{1,2}(\Omega)$ the Sobolev space with the usual norm $\|\cdot\|_{2}=\|\cdot\|_{W_{0}^{1,2}(\Omega)}$. Let also $p$ and $\varepsilon$ such that $0<\varepsilon<1$, $\varepsilon+2<p<\infty$. Set $\|\cdot\|_{1}=\|\cdot\|_{L^{p}(\Omega)}$. Note that $\left(E,\|\cdot\|_{2}\right)$ is a separable Banach space.

Since $W_{0}^{1,2}(\Omega) \hookrightarrow L^{p}(\Omega)$, it follows that $\left(E,\|\cdot\|_{1},\|\cdot\|_{2}\right)$ is a binormed space such that $\|\cdot\|_{1} \leq c\|\cdot\|_{2}$ for some $c>0$. 
Set $g(u)=|u|^{\varepsilon+2}$ and consider the functional $G$ defined on $E$ by

$$
G(x)=\int_{\Omega} g(x(s)) d s .
$$

Then $G$ is $\|\cdot\|_{2}$ twice Fréchet differentiable at every $x \in E$ and

$$
\begin{aligned}
G^{(1)}(x) h & =\int_{\Omega} g^{\prime}(x(s)) h(s) d s, \\
G^{(2)}(x)\left(h_{1}, h_{2}\right) & =\int_{\Omega} g^{\prime \prime}(x(s)) h_{1}(s) h_{2}(s) d s .
\end{aligned}
$$

We assert that $G$ is $\left(\|\cdot\|_{1},\|\cdot\|_{2}\right)$ strictly differentiable at every $x \in E$. Indeed, suppose by the contrary that there exist $x \in E, \varepsilon>0, x_{m}^{\prime} \in E$, and $h_{m} \in E$ such that $x_{m}^{\prime} \rightarrow x$ in $\left(E,\|\cdot\|_{1}\right), h_{m} \rightarrow 0$ in $\left(E,\|\cdot\|_{1}\right)$ and $\left|r\left(x_{m}^{\prime}, h_{m}\right)\right|>\varepsilon\left\|h_{m}\right\|_{2}$, where

$$
r\left(x^{\prime}, h\right)=G\left(x^{\prime}+h\right)-G\left(x^{\prime}\right)-G^{(1)}(x) h .
$$

Then,

$$
\left|r\left(x_{m}^{\prime}, h_{m}\right)\right|=\left|\int_{\Omega} \int_{0}^{1}\left(g^{\prime}\left(x_{m}^{\prime}(s)+t h_{m}(s)\right)-g^{\prime}(x(s))\right) h_{m}(s) d t d s\right| .
$$

Let $p^{\prime}>1$ such that $\frac{1}{p}+\frac{1}{p^{\prime}}=1$. Since $p>\varepsilon+2$ then $p>p^{\prime}(\varepsilon+1)$. Thus, $x_{m}^{\prime} \rightarrow x$ in $\left(E,\|\cdot\|_{L^{p^{\prime}(\varepsilon+1)}(\Omega)}\right)$ and $h_{m} \rightarrow 0$ in $\left(E,\|\cdot\|_{L^{p^{\prime}(\varepsilon+1)}(\Omega)}\right)$. Without loss of generality we can suppose that there exists $Z_{1} \in L^{p^{\prime}(\varepsilon+1)}(\Omega)$ such that

$$
\left|x_{m}^{\prime}(s)\right|+\left|h_{m}(s)\right| \leq Z_{1}(s), \quad h_{m}(s) \rightarrow 0, \quad x_{m}^{\prime}(s) \rightarrow x(s)
$$

almost everywhere on $\Omega$. Using the Hölder inequality, we obtain

$$
\left|r\left(x_{m}^{\prime}, h_{m}\right)\right| \leq\left(\int_{\Omega} \int_{0}^{1}\left|g^{\prime}\left(x_{m}^{\prime}(s)+t h_{m}(s)\right)-g^{\prime}(x(s))\right|^{p^{\prime}} d t d s\right)^{\frac{1}{p^{\prime}}}\left\|h_{m}\right\|_{L^{p}(\Omega)} .
$$

Consequently,

$$
\left|r\left(x_{m}^{\prime}, h_{m}\right)\right| \leq c\left(\int_{\Omega} \int_{0}^{1}\left|g^{\prime}\left(x_{m}^{\prime}(s)+t h_{m}(s)\right)-g^{\prime}(x(s))\right|^{p^{\prime}} d t d s\right)^{\frac{1}{p^{\prime}}}\left\|h_{m}\right\|_{2} .
$$

But this contradicts the fact that $\left|r\left(x_{m}^{\prime}, h_{m}\right)\right|>\varepsilon\left\|h_{m}\right\|_{2}$, because

$$
\int_{\Omega} \int_{0}^{1}\left|g^{\prime}\left(x_{m}^{\prime}(s)+t h_{m}(s)\right)-g^{\prime}(x(s))\right|^{p^{\prime}} d t d s \rightarrow 0,
$$

by the dominated convergence theorem.

Let us remark that $G$ is not $\|\cdot\|_{1}$ Fréchet differentiable at any point $x \in E$. Indeed, let $\alpha_{m} \rightarrow \infty$ and $d_{m} \rightarrow \infty$ such that $\left|g\left(d_{m}\right)\right| \geq \alpha_{m}\left|d_{m}\right|^{p}$. By the countable additivity of the Lebesgue measure there exist $C>0$ and $\Omega^{\prime} \subset \Omega$ such that $\mu\left(\Omega^{\prime}\right)>0$, $\operatorname{dist}\left(\Omega^{\prime}, \partial \Omega\right)>0$, and $|x(s)| \leq C$ for all $s \in \Omega^{\prime}$. 
In this case, put $D=\max \{g(u):|u| \leq C\}<\infty$. Choose $\Omega_{m} \subset \Omega^{\prime}$ such that $\mu\left(\Omega_{m}\right)=\left|d_{m}\right|^{-p}\left|\alpha_{m}\right|^{-\frac{1}{2}}$ for large $m$.

Let $h_{m}$ be defined by the relation

$$
h_{m}(s)= \begin{cases}d_{m}-x(s) & \text { for } \quad s \in \Omega_{m}, \\ 0 & \text { for } \quad s \in \Omega \backslash \Omega_{m} .\end{cases}
$$

It then follows that $\left\|h_{m}\right\|_{1} \rightarrow 0$ and

$$
\left|G\left(x+h_{m}\right)-G(x)\right| \geq \alpha_{m}\left|d_{m}\right|^{p} \mu\left(\Omega_{m}\right)-D \mu\left(\Omega_{m}\right)=\left|\alpha_{m}\right|^{\frac{1}{2}}-D \mu\left(\Omega_{m}\right) \rightarrow \infty .
$$

Let $k_{m} \in C_{0}^{\infty}(\Omega)$ such that $\left\|k_{m}-h_{m}\right\|_{1} \rightarrow 0$. Then $\left\|k_{m}\right\|_{1} \rightarrow 0$, but $G\left(x+k_{m}\right)-$ $G(x) \rightarrow \infty$, because the Lebesgue integral is absolutely continuous. Therefore, $G$ is not $\|\cdot\|_{1}$ Fréchet differentiable at $x$ and consequently, $G$ is not $\|\cdot\|_{1}$ strictly Fréchet differentiable at $x$.

Thus, applying Theorem 1, we conclude that $\partial_{0}^{1,2} G(x)=\partial_{0}^{2} G(x)=\{\nabla G(x)\}$.

\section{REFERENCES}

[1] J. M. Borwein and A. S. Lewis, Convex analysis and nonlinear optimization, ser. CMS Books in Mathematics/Ouvrages de Mathématiques de la SMC, 3. New York: Springer-Verlag, 2000, theory and examples.

[2] F. H. Clarke, Y. S. Ledyaev, R. J. Stern, and P. R. Wolenski, Nonsmooth analysis and control theory, ser. Graduate Texts in Mathematics. New York: Springer-Verlag, 1998, vol. 178.

[3] F. H. Clarke, "Generalized gradients and applications," Trans. Amer. Math. Soc., vol. 205, pp. $247-$ 262, 1975.

[4] F. H. Clarke, Optimization and nonsmooth analysis, ser. Canadian Mathematical Society Series of Monographs and Advanced Texts. New York: John Wiley \& Sons Inc., 1983, a Wiley-Interscience Publication.

[5] S. Lahrech and A. Benbrik, "On the Mordukhovich subdifferential in binormed spaces and some applications," Int. J. Pure Appl. Math., vol. 20, no. 1, pp. 31-39, 2005.

[6] P. Michel and J.-P. Penot, "Calcul sous-différentiel pour des fonctions lipschitziennes et non lipschitziennes,” C. R. Acad. Sci. Paris Sér. I Math., vol. 298, no. 12, pp. 269-272, 1984.

Author's address

\section{S. Lahrech}

Department of Mathematics, Faculty of Science, Mohamed First University, Oujda, Morocco

E-mail address: lahrechesciences.univ-oujda.ac.ma 